\title{
MLC901 for Moderate to Severe Traumatic Brain Injury: Pilot, Randomized, Double-Masked, Placebo- Controlled Trial
}

\author{
Hossein Pakdaman, Ali Amini Harandi*, Akram Esfandani, Atefeh Yousefi, Marziyeh Khazai Pol, Behnam Mansouri, \\ Ali Sobhanian and Sina Asadi
}

Brain Mapping Research Center, Shahid Beheshti University of Medical Sciences, Tehran, Iran

*Corresponding author: Ali Amini Harandi, Brain Mapping Research Center, Shahid Beheshti University of Medical Sciences, South Kargar Street, Tehran, Iran

\begin{abstract}
Background: Despite recent developments in intensive care for patients with Traumatic brain injury (TBI), the long-term neurological disabilities still exist. MLC901 is Traditional Chinese Medicine and has shown some neuroprotective and neuro regenerative benefits after brain injury in previous animal and human studies and may provide a new therapeutic approach in the treatment of TBI. Accordingly, we conducted this pilot, randomized, double-masked, placebo-controlled study to investigate the efficacy of MLC901 in patients with moderate to severe TBI.
\end{abstract}

Materials and Methods: Patients with a diagnosis of moderate to severe TBI were enrolled. Subjects were randomly assigned to receive either MLC901 or placebo capsules three times per day over 6 months. Evaluation of patients was carried out at baseline, $3^{\text {rd }}$ month and $6^{\text {th }}$ month follow-after injury. Modified Rankin Scale (mRS) and Glasgow outcome scale (GOS) were used to examine patients. Efficacy was evaluated by comparing these two scores between the 2 groups at follow-up visits.

Results: Eighty-one patients complete 6 months follow up. There was no significant difference between two study groups regarding the demographic features, interval between injury and start of intervention and length of ICU stay. However, functional outcome scales of GOS, MRS at $3^{\text {rd }}$ and $6^{\text {th }}$ month post-injury were significantly better in MLC901 group compared to placebo $(\mathrm{p}<0.05)$.

Conclusion: MLC901 has shown promising efficacy in patients suffering from moderate to severe TBI.

Keywords: NeuroAid, MLC901; Traumatic brain injury; Placebo-controlled study, Modified rankin scale, Glasgow outcome scale, Functional outcome

\section{Introduction}

Traumatic brain injury (TBI) has significant morbidity, mortality and is the major cause of disability in individuals younger than 35 years in the Europe and the USA. Traumatic brain injury (TBI) is also one of the causes of epilepsyanda substantial proportion of traumatic deaths are related to TBI [1]. TBI with its large socioeconomic consequences and its high incidence in young adults is still an important health concern worldwide and poses a real challenge to public health. Despite recent developments in intensive care for patients with TBI, long-term neurological disabilities related to TBI still exist. More than half of patients show significant after-effects, principally in cognitive tasks. Neuroprotective therapeutic Studies have still not introduced safe, and effective treatment for TBI [2,3]. MLC901 (Neuro Aid) as Traditional Chinese Medicine could provide a new therapeutic approach in the treatment of TBI. Therapeutic advantages of MLC901 have already been depicted in patients suffering from stroke, Alzheimer's disease, mild cognitive impairment and vascular dementia [4-6]. Consistent with findings of clinical reports in efficacy and safety of MLC901, animal studies 
have also established that MLC901 prevents the death of neuronal tissues, decreases cognitive dysfunction and also improves functional neurological outcome by restoring neuronal circuits in models of ischemia. MLC901has also shown neuroprotective and neurorestorative actions, which could potentially lead to the enhanced recovery of cognitive functions in animal models of TBI. The efficacy of MLC901 has also been shown the oxygenglucose deprivation model, which mimics ischemic conditions $[7,8]$. Additionally, in a recent study, MLC901 has demonstrated to improve cognitive functioning in patients with mild to moderate TBI [9]. In this study, we aimed to evaluate the effect of MLC901 on neurological function and outcome in patients suffering from moderate to severe TBI.

\section{Materials and Methods}

\section{Study design and patients}

This was a randomized, double-blind, Placebo-controlled, study conducted between May 2017 and December 2019. The aim of the study was to determine efficacy of MLC901 on neurological outcome after injury in patients with moderate or severe TBI. Clinicians recruited patients from University-affiliated hospitals and patients were eligible for this study if they satisfied the following inclusion criteria: age between 15 and 65, nonpenetrating moderate (Glasgow Coma Scale score [GCS] 9-12) or severe (GCS 3-8) traumatic brain injury less than 24 hours from traumatic injury, anticipated intensive care unit length of stay at least 48 hours. Patients were excluded if GCS was 3 and they had fixed dilated pupils or penetrating injury, coexisting injury or medical conditions which could adversely affect our study outcome measures, dependence for everyday activities before the injury, pregnancy or breastfeeding, known allergy to any of MLC901 components.

MLC901 contains nine herbal components $(0.114 \mathrm{~g}$ radix Paeoniae rubrae, $0.57 \mathrm{~g}$ radix Astragali, $0.114 \mathrm{~g}$ radix Salviae miltiorrhizae, 0.114g rhizoma Chuanxiong, 0.114g radix Angelicae sinensis, 0.114g radix Polygalae, 0.114g Prunus persica, $0.114 \mathrm{~g}$ Carthamus tinctorius, and 0.114g rhizoma Acori tatarinowii) [10].

Patients were randomized to either MLC901 or placebo group with a simple allocation ratio of 1:1. Randomization was stratified based on age, gender, baseline and GCS. Patients received either MLC901 or visually indistinguishable placebo capsules (vegetable capsules that filled by less than 2 grams of stevia sweetened powder) for 6 months supply. The dose was two capsules $(0.4 \mathrm{~g} /$ capsule) taken orally three times per day for 6 months. Patients were evaluated 3 month and 6 months after treatment initiation.

\section{Outcome measures}

Modified Rankin Scale (mRS) and Glasgow outcome scale (GOS) were utilized to examine participants at the baseline, 3 and 6-month follow-up visits. mRS is a widely used scale to determine the level of disability in patients with neurological disorders. It ranges between 0 and 6 with a higher score indicating a higher degree of disability [11] (Table 1). GOS is also another commonly used scale to evaluate patients with brain injuries such as TBI. This scale ranges between 1 and 5 with the highest score being the good recovery [12] (Table 1). All evaluations were conduct by medical staff who were unaware about randomization of patients in each group.

Table 1: Modified Rankin Scale (mRS) and Glasgow Outcome Scale (GOS) subscales.

\begin{tabular}{|c|c|c|c|}
\hline \multicolumn{2}{|l|}{ mRS } & \multicolumn{2}{|l|}{ GOS } \\
\hline No symptoms & $\mathbf{0}$ & Death & 1 \\
\hline $\begin{array}{l}\text { No significant disability. Despite some symptoms, able to carry } \\
\text { out all usual activities }\end{array}$ & 1 & & \\
\hline $\begin{array}{l}\text { Slight disability. Able to look after own affairs without } \\
\text { assistance, but unable to carry out all previous activities. }\end{array}$ & 2 & Persistent vegetative state: Minimal responsiveness & 2 \\
\hline $\begin{array}{l}\text { Moderate disability. Able to walk without assistance but } \\
\text { requires some help. }\end{array}$ & 3 & $\begin{array}{l}\text { Severe disability: Conscious but disabled; dependent on } \\
\text { others for daily support }\end{array}$ & 3 \\
\hline $\begin{array}{l}\text { Moderately severe disability. Unable to walk unassisted. } \\
\text { Unable to attend to own bodily needs without assistance. }\end{array}$ & 4 & $\begin{array}{l}\text { Moderate disability: Disabled but independent; can work in } \\
\text { sheltered setting }\end{array}$ & 4 \\
\hline $\begin{array}{l}\text { Severe disability. Incontinent, bedridden. Requires constant } \\
\text { nursing care and attention. }\end{array}$ & 5 & $\begin{array}{c}\text { Good recovery: Resumption of normal life despite minor } \\
\text { deficits }\end{array}$ & 5 \\
\hline Dead & 6 & & \\
\hline
\end{tabular}

\section{Statistical analysis}

All statistical analysis was done by SPSS (version 16.0) software [Statistical Procedures for Social Sciences; Chicago, Illinois, USA] with the intention to treat approach. Descriptive data were reported as a mean \pm standard deviation for Continuous values and frequency for categorical values. Normal distributions of values were determined by the Kolmogorov-Smirnov test. Non-parametric data were compared using chi-square test. Kruskal-Wallis test was used to compare parametric data without normal distribution between two study groups. P values less than 0.05 were considered a statistically significant. 


\section{Ethical issues}

Ethic committee of Shahid Beheshti University of Medical Science, Tehran, Iran approved all study protocols and written consent provided by each patient's legal surrogate according to
Helsinki Declaration and as permitted by local regulations. The protocol has been registered (ClinicalTrials.gov ID: NCT04487275). Patients who recovered with decision-making capacity were asked to consent to continue participating in the study.

\section{Results}

Table 2: Baseline characteristics.

\begin{tabular}{|c|c|c|c|}
\hline Parameter & MLC901 (n=44) & Placebo (n=37) & P value \\
\hline Age & $39.98( \pm 14.4)$ & $40.63( \pm 15.1)$ & 0.49 \\
\hline Gender (males) & $24(54.5 \%)$ & $22(59.4 \%)$ & 0.82 \\
\hline Injury to intervention (hr)* & $7.76( \pm 7.51)$ & $7.09( \pm 3.61)$ & 0.62 \\
\hline Length of ICU stay (day) & $12.66( \pm 9.56)$ & $13.53( \pm 9.28)$ & 0.68 \\
\hline
\end{tabular}

*Interval between injury and start of intervention.

ICU: intensive care unit.

Table 3: The modified Rankin Scale of patients with moderate and severe traumatic brain injury being treated with MLC901 vs. placebo.

\begin{tabular}{|c|c|c|c|c|c|c|c|c|c|c|}
\hline \multicolumn{2}{|c|}{ mRS } & \multirow{2}{*}{$\begin{array}{c}\text { Mean } \\
2.06 \pm 0.8\end{array}$} & \multirow{2}{*}{$\begin{array}{c}\text { No } \\
\text { Symptoms }\end{array}$} & \multirow{2}{*}{$\begin{array}{c}\text { No } \\
\text { significant } \\
\text { disability }\end{array}$} & \multirow{2}{*}{$\begin{array}{c}\begin{array}{c}\text { Slight } \\
\text { disability }\end{array} \\
13(29.5)\end{array}$} & \multirow{2}{*}{$\begin{array}{c}\begin{array}{c}\text { Moderate } \\
\text { disability }\end{array} \\
1(2.3)\end{array}$} & \multirow{2}{*}{$\begin{array}{c}\text { Moderately } \\
\text { severe } \\
\text { disability }\end{array}$} & \multirow{2}{*}{$\begin{array}{c}\begin{array}{c}\text { Severe } \\
\text { disability }\end{array} \\
0\end{array}$} & \multirow{2}{*}{$\begin{array}{c}\text { Dead } \\
0 \\
\end{array}$} & \multirow{2}{*}{$\begin{array}{r}\text { P-value } \\
0.024\end{array}$} \\
\hline & MLC901 & & & & & & & & & \\
\hline 3 month & Placebo & $2.40 \pm 0.9$ & $5(13.5)$ & $19(51.4)$ & $6(16.6)$ & $7(18.9)$ & 0 & 0 & 0 & \\
\hline \multirow{2}{*}{$6^{\text {th }}$ months } & MLC901 & $1.90 \pm 0.7$ & $14(31.8)$ & $20(45.5)$ & $10(22.7)$ & 0 & 0 & 0 & 0 & 0.04 \\
\hline & Placebo & $2.27 \pm 0.7$ & $4(10.8)$ & $22(59.5)$ & $8(21.6)$ & $3(8.1)$ & 0 & 0 & 0 & \\
\hline
\end{tabular}

Table 4: The Glasgow Outcome Scale of patients with moderate and severe traumatic brain injury being treated with MLC901 vs. placebo.

\begin{tabular}{|c|c|c|c|c|c|c|c|c|}
\hline \multicolumn{2}{|c|}{ GOS } & Mean & Dead & $\begin{array}{l}\text { Persistent } \\
\text { vegetative }\end{array}$ & $\begin{array}{c}\text { Severe } \\
\text { disability }\end{array}$ & $\begin{array}{l}\text { Moderate } \\
\text { disability }\end{array}$ & $\begin{array}{l}\text { Good } \\
\text { recovery }\end{array}$ & P-value \\
\hline \multirow{2}{*}{$3^{\text {rd }}$ month } & MLC901 & $3.95 \pm 0.8$ & 0 & $3(6.8)$ & $9(20.5)$ & $19(43.2)$ & $13(29.5)$ & \multirow{2}{*}{0.023} \\
\hline & Placebo & $3.40 \pm 0.8$ & 0 & $4(10.8)$ & $18(48.6)$ & 11(29.7) & $4(10.8)$ & \\
\hline \multirow{2}{*}{$6^{\text {th }}$ month } & MLC901 & $4.29 \pm 0.7$ & 0 & 0 & $6(13.6)$ & $19(43.2)$ & $19(43.2)$ & \multirow{2}{*}{0.006} \\
\hline & Placebo & $3.75 \pm 0.7$ & 0 & 0 & $16(43.2)$ & $14(37.8)$ & $7(18.9)$ & \\
\hline
\end{tabular}

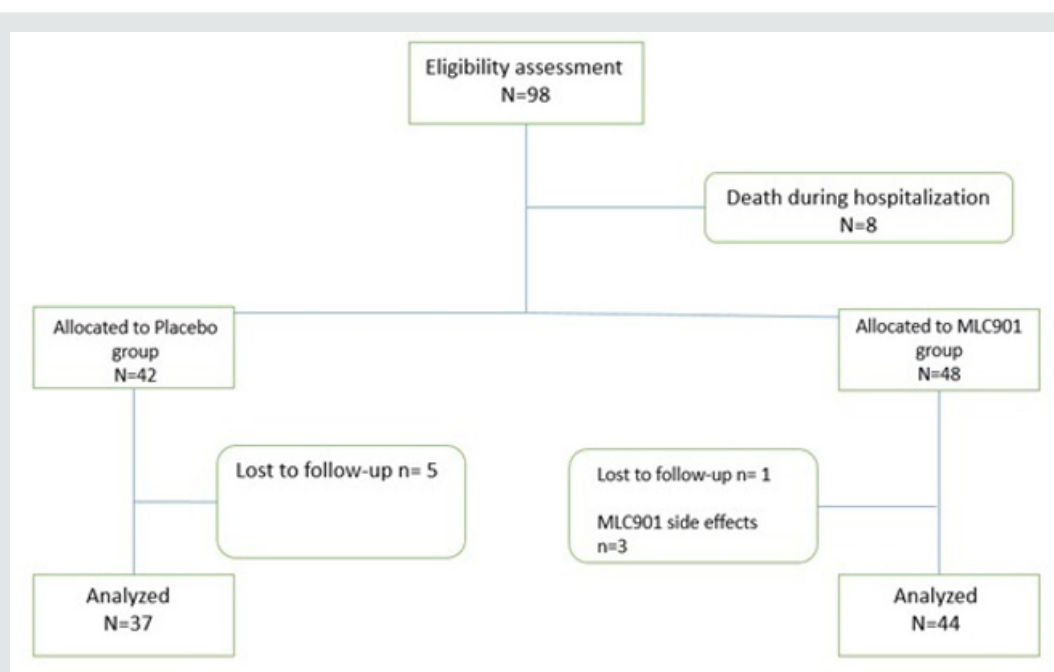

Figure 1: CONSORT flow diagram of the study. 
Totally 98 Patients recruited in this study. Seventeen patients were excluded from the study due to occurrence of MLC901 side effect $(n=3)$, loss of follow-up $(n=6)$, and death during hospitalization $(n=8)$. The CONSORT flow diagram of the study is demonstrated in figure 1. Baseline patients' characteristics, interval between injury and start of intervention, and length of intensive care unit (ICU) stay in both groups were not significantly different between the two study groups (Table 2). The comparison of mRS and GOS scores between the two groups are depicted in Tables 3 $\& 4$.

\section{Discussion}

Our primary findings provide evidence that MLC901 helps in the recovery of patients with moderate to severe TBI. There were significant differences between the two groups in mRS and GOS scores at follow-up visits. Improvement of these two scales suggests the potential usefulness of MLC901 at 6 months period after injury. The brain is highly plastic following TBI and improvement in function can be observed over a year after initial injury, therefore, initiation of MLC901 during natural recovery period can facilitate this process. In our study, MLC901 was given within first 24 hours after TBI and it is likely that anti-inflammation and antioxidant properties of MLC901 have affected recovery in its initial phase and current evidence suggest the efficacy of these properties over the first-year post-injury $[13,14]$.

Various studies showed effect of different medication on TBI based on mRS and GOS scores. Farzanegan et al, showed that, functional outcome scales of GOS, mRS and at 3-months post-injury were significantly better in patients with TBI who used atorvastatin in compare to placebo group [15]. In another study by Khalili et al, 3-months assessment of outcome by mRS and GOS showed no significant different in patients who received glibenclamide in compare with placebo group [16]. To the best of our knowledge, this is the first study evaluating the efficacy of MLC901 in moderate to severe TBI, consequently direct comparison of our findings to previous studies is not possible. However, there is one study that has reported the efficacy of MLC901 in mild to moderate TBI. In the study by Theadon et al, authors evaluated the efficacy of MLC901 on cognition in 78 patients suffering from mild to moderate TBI over 9 months and they used multiple questionnaires and scales to assess participants in 1 to 12 months after TBI. They reported significant improvements in complex attention and executive functioning They also found that MLC901 has no advantage over placebo in terms of neurobehavioral sequelae, mood, fatigue, physical disability or overall quality of life. No serious adverse events were also reported [9]. Their findings are partially in line with the results of a current study in this way that MLC901 is an effective therapy for TBI regardless of its severity.
It is now well accepted that the functional recovery that occurs spontaneously after brain injury (stroke or TBI) is due to the plasticity of surviving neurons, lesion-induced plasticity, and/or plasticity of neural connections $[17,18]$. In the other hand, overall cellular remodeling that occurs after traumatic brain injury results to proliferation of newly generated cells [19]. Although there is a lack of clinical studies, experimental studies on TBI shows that both gliogenesis and neurogenesis induced by MLC901, probably help in inducing a dynamic brain remodeling and lead to a better neurologic recovery in the first weeks after TBI $[18,19]$. In animal model studies, Quintard et al. evaluated the neuroprotective and neuro regenerative effects of MLC901 in a rat model of TBI. In their study MLC901 inhibited the increase of S-100 beta and neuronspecific enolase in serum, that are possible predictor markers of neurologic outcome in patients with TBI. Also, they showed that probably regulation of aquaporin 4 by injection of MLC901 2 hour after TBI resulted to infarct volume reduction, prevented edema formation and assisted its resolution [20]. In addition, recently animal studies on focal and global ischemia have approved effects of MLC901 in increased neurogenesis, neurite outgrowth, axonal sprouting, dendritic arborization and/or synaptogenesis that are correlated with functional recovery [7,21].

Neuroprotective effects of MLC901 in ischemic brain injuries was suggested through activation of Toll-like receptor 4 (TLR4) signaling and inhibition of the expression of Prx6 and the transcriptional activity of $N F \kappa B$ which are main pathway in the immune system response to injury [10]. Quintard et al, also reported that in animal models of TBI, MLC901 effect around the lesion included vascular endothelial growth factor (VEGF) upregulation, hippocampal neurogenesis and gliogenesis [20]. Brain-derived neurotrophic factor (BDNF)and VEGF are well-known growth promoting factors that play a key role to support adaptive remodeling of surviving neurons and neural networks modulation in recovery process $[22,23]$. VEGF is an important tissue repair mediator after brain injury and has substantial role in angiogenesis [24]. In the other hand BDNF upregulation by MLC901 has been reported in brain injuries due to stroke and cardiac arrest [7,22,25]. It seems that increased expression of VEGF and BDNF is another positive effect of MLC901 that could be responsible for the findings that observed in this study.

The combination of multiple herbal components is thought to maximize therapeutic efficacy by facilitating synergistic actions and ameliorating or preventing potential adverse effects while at the same time aiming at multiple targets [22]. In the neuroinflammation study by Widman and Heurteaux list assumptions of molecules which may explain part of the effects $[10,22]$. In these different herbs that are extracted to manufacture MLC901, there are a number of recognized molecules such as ferulic acid ferulic acid 
(Radix angelicae sinensis, Rhizoma chuanxiong) [26], salvianolic acid B and tanshinone IIB (Radix salvia miltiorrhizae) [27-28] tetramethylpyrazine (Rhizoma chuanxiong) [29-30], ligustilide and butylidenephtalide (Radix angelicae sinensis, Rhizoma chuanxiong) [31-32], astragaloside IV (Radix astragali) [33], ligustilide and butylidenephtalide (Radix angelicae Sinensis, salvianolic acid B and tanshinone IIB (Radix salvia miltiorrhizae) that are identified for neurobeneficial and anti-inflammatory activity $[10,23]$.

There are several strengths as well as limitations to our study. Recruitments of patients were challenging since their surrogates or patients mentioned taking capsules three times daily over 6 months as a hurdle to participate in the study and some suspect this many capsules could harm patients rather than help them in recovery. Probably, decreasing the daily dosage administration would help in participation and adherence of patients. Additionally, moderate to severe TBI is known to be related to cognitive deficits, therefore, aids to support memory may increase patient's adherence and compliance. We used GOS to assess patient's outcome. Recently is suggested that Glasgow Outcome Scale-Extended (GOSE) has priority to the GOS. GOSE in extended version of GOS that improve sensitivity of minor changes detection in functional activity [34]. Our study also suffers from a limited number of patients. Lack of patients self-reporting questionnaire is another issue with this study. We didn't measure any neuroplasticity biomarkers in our patients, but it seems that measuring the neuroplasticity biomarkers in future studies could be useful to understand the underlying effects of MLC901 in TBI. We suggest, with the current promising result of the present study, more studies with a larger group of patients with different evaluation scales and longer duration of follow-up should be performed to draw a better conclusion.

\section{Conclusion}

This work provides evidence that MLC901 is an effective therapeutic approach in patients suffering from moderate to severe TBI. However further studies with more clinical and paraclinical assessments are suggested to evaluate different aspects of MLC901effectiveness in neurologic disease and particularly TBI.

\section{Acknowledgments}

None.

\section{Conflict of Interest}

None.

\section{References}

1. Bruns J Jr, Hauser WA (2003) The epidemiology of traumatic brain injury: a review. Epilepsia 44(s10): 2-10.

2. Ruttan L, Martin K, Liu A (2008) Long-term cognitive outcome in moderate to severe traumatic brain injury: a meta-analysis examining timed and untimed tests at 1 and 4.5 or more years after injury. Arch Phys Med Rehabil 89(12suppl): S69-S76.
3. Xiong Y, Mahmood A, Chopp M (2009) Emerging treatments for traumatic brain injury. Expert Opin Emerg Drugs 14(1): 67-84.

4. Pakdaman H, Amini Harandi A, Gharagozli K, Mehdi Abbasi, Majid Ghaffarpour, et al. (2017) MLC601 in vascular dementia: an efficacy and safety pilot study. Neuropsychiatr Dis Treat 13: 2551-2557.

5. Pakdaman H, Amini Harandi A, Abbasi M, Delavar Kasmaei H, Ashrafi F, et al. (2017) Efficacy and Safety of MLC601 in the Treatment of Mild Cognitive Impairment: A Pilot, Randomized, Double-Blind, PlaceboControlled Study. Dementia and Geriatric Cognitive Disorders Extra 7(1): 136-142.

6. Pakdaman H, Gharagozli K, Abbasi M, Ali Sobhanian, Ali Bakhshandehpour, et al. (2018) Efficacy and Safety of MLC601 in Patients with Mild to Moderate Alzheimer Disease: An Extension 4-Year Follow-Up Study. Dement Geriatr Cogn Dis Extra 8(1): 174-179.

7. H Quintard, M Borsotto, J Veyssiere, C Gandin, F Labbal, et al. (2011) MLC901, a traditional Chinese medicine protects the brain against global ischemia. Neuropharmacology 61(4): 622-631.

8. H Moha Ou Maati, M Borsotto, F Chatelain, C Widmann, M Lazdunski, et al. (2012) Activation of ATP-sensitive potassium channels as an element of the neuroprotective effects of the Traditional Chinese Medicine MLC901 against oxygen glucose deprivation. Neuropharmacology 63(4): 692-700

9. A Theadom, S Barker-Collo, K M Jones, $\mathrm{P}$ Parmar, $\mathrm{R}$ Bhattacharjee, et al. (2018) MLC901 (NeuroAiD II $^{\mathrm{TM}}$ ) for cognition after traumatic brain injury: a pilot randomized clinical trial. Eur J Neurol 25(8): 1055-e1082.

10. Widmann C, Gandin C, Petit-Paitel A, M Lazdunski, C Heurteaux (2018) The Traditional Chinese Medicine MLC901 inhibits inflammation processes after focal cerebral ischemia. Sci Rep 8:18062.

11. Sadaka F, Patel D, Lakshmanan R (2012) The FOUR score predicts outcome in patients after traumatic brain injury. Neurocrit Care 16(1): 95-101.

12. Hall K, Cope DN, Rappaport M (1985) Glasgow Outcome Scale and Disability Rating Scale: comparative usefulness in following recovery in traumatic head injury. Arch Phys Med Rehabil 66(1): 35-37.

13. Rigg JL, Elovic EP, Greenwald BD (2005) A review of the effectiveness of antioxidant therapy to reduce neuronal damage in acute traumatic brain injury. J Head Trauma Rehabil 20(4): 389-391.

14. Suzanne Barker-Collo, Kelly Jones, Alice Theadom, Nicola Starkey, Anthony Dowell, et al. (2015) Neuropsychological outcome and its correlates in the first year after adult mild traumatic brain injury: A population-based New Zealand study. Brain Inj 29(13-14): 1604-1616.

15. Gholam Reza Farzanegan, Nima Derakhshan, Hosseinali Khalili, Fariborz Ghaffarpasand, Shahram Paydar (2017) Effects of atorvastatin on brain contusion volume and functional outcome of patients with moderate and severe traumatic brain injury; a randomized double-blind placebocontrolled clinical trial. J Clin Neurosci 44: 143-147.

16. Hosseinali Khalili, Nima Derakhshan, Amin Niakan, Fariborz Ghaffarpasand, Mohammad Salehi, et al. (2017) Effects of Oral Glibenclamide on Brain Contusion Volume and Functional Outcome of Patients with Moderate and Severe Traumatic Brain Injuries: A Randomized Double-Blind Placebo-Controlled Clinical Trial. World Neurosurg 101: 130-136

17. Murphy TH, Corbett D (2009) Plasticity during stroke recovery: from synapse to behaviour. Nat Rev Neurosci 10(12): 861-872.

18. Schoch KM, Madathil SK, Saatman KE (2012) Genetic manipulation of cell death and neuroplasticity pathways in traumatic brain injury. Neurotherapeutics 9(2): 323-337.

19. R Mark Richardson, Amanpreet Singh, Dong Sun, Helen L Fillmore, Dalton W Dietrich $3^{\text {rd }}$, et al. (2010) Stem cell biology in traumatic brain injury: effects of injury and strategies for repair. J Neurosurg 112(5): 1125-1138. 
20. H Quintard, T Lorivel, C Gandin, M Lazdunski, C Heurteaux (2014) MLC901, a Traditional Chinese Medicine induces neuroprotective and neuroregenerative benefits after traumatic brain injury in rats. Neuroscience 277: 72-86.

21. C Heurteaux, C Gandin, M Borsotto, C Widmann, F Brau, et al. (2010) Neuroprotective and neuroproliferative activities of NeuroAid (MLC601, MLC901), a Chinese medicine, in vitro and in vivo. Neuropharmacology 58(7): 987-1001.

22. Conte V, Royo NC, Shimizu S (2003) Neurotrophic Factors. Eur J Trauma 29: 335-355.

23. Chopp M, Li Y, Zhang ZG (2009) Mechanisms underlying improved recovery of neurological function after stroke in the rodent after treatment with neurorestorative cell-based therapies. Stroke $40(3$ Suppl): S143-S145.

24. Greenberg DA, Jin K (2005) From angiogenesis to neuropathology. Nature 438(7070): 954-959.

25. Heurteaux C, Widmann C, Moha ou Maati H (2013) NeuroAiD: Properties for Neuroprotection and Neurorepair. Cerebrovasc Dis 35:1-7.

26. Chin-Yi Cheng, Tin-Yun Ho, E-Jian Lee, Shan-Yu Su, Nou-Ying Tang, et al. (2008) Ferulic acid reduces cerebral infarct through its antioxidative and anti-inflammatory effects following transient focal cerebral ischemia in rats. Am J Chin Med 36(6):1105-1119.

27. Shao-Xia Wang, Li-Min Hu, Xiu-Mei Gao, Hong Guo, Guan-Wei Fan (2010) Anti-inflammatory activity of salvianolic acid B in microglia contributes to its neuroprotective effect. Neurochem Res 35(7): 1029-1037.
28.Xi-Yong Yu, Shu-Guang Lin, Zhi-Wei Zhou, Xiao Chen, Jun Liang, et al. (2007) Tanshinone IIB, a primary active constituent from Salvia miltiorrhza, exhibits neuro-protective activity in experimentally stroked rats. Neurosci Lett 417(3): 261-265.

29. Tsung-Kuei Kao, Yen-Chuan Ou, Jong-Song Kuo, Wen-Yin Chen, Su-Lan Liao, et al. (2006) Neuroprotection by tetra methylpyrazine against ischemic brain injury in rats. Neurochem Int 48(3): 166-176.

30. Su-Lan Liao, Tsung-Kuei Kao, Wen-Yin Chen, Yu-Sheng Lin, Shih-Yun Chen, et al. (2004) Tetramethylpyrazine reduces ischemic brain injury in rats. Neurosci Lett 372(1-2): 40-45.

31. Xi Kuang, Jun-Rong Du, Yan-Xin Liu, Guang-Yi Zhang, Hai-Yan Peng (2008) Postischemic administration of Z-Ligustilide ameliorates cognitive dysfunction and brain damage induced by permanent forebrain ischemia in rats. Pharmacol Biochem Behav 88(3): 213-221.

32. Song-Jie Liao, Jian-Wen Lin, Zhong Pei, Chun-Ling Liu, Jin-Sheng Zeng, et al. (2009) Enhanced angiogenesis with dl-3n-butylphthalide treatment after focal cerebral ischemia in RHRSP. Brain Res 1289: 69-78.

33. Yumin Luo, Zhen Qin, Zhen Hong, Xinmin Zhang, Ding Ding, et al. (2004) Astragaloside IV protects against ischemic brain injury in a murine model of transient focal ischemia. Neurosci Lett 363(3): 218-223.

34. James Weir, Ewout W Steyerberg, Isabella Butcher, Juan Lu, Hester F Lingsma, et al. (2012) Does the extended Glasgow Outcome Scale add value to the conventional Glasgow Outcome Scale. J Neurotrauma 29(1): 53-58.

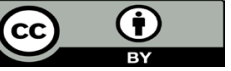

This work is licensed under Creative Commons Attribution 4.0 License

To Submit Your Article Click Here:

Submit Article
DOI: 10.32474/OAJCAM.2020.02.000148

Citation: Hossein Pakdaman, Ali Amini Harandi, Akram Esfandani, Atefeh Yousefi, Marziyeh Khazai Pol, et al., MLC901 for Moderate to Severe Traumatic Brain Injury: Pilot, Randomized, Double-Masked, Placebo-Controlled Trial. Open Acc J Comp \& Alt Med 2(5) - 2020. OAJCAM.MS.ID.000148. DOI: 10.32474/OAJCAM.2020.02.000148.

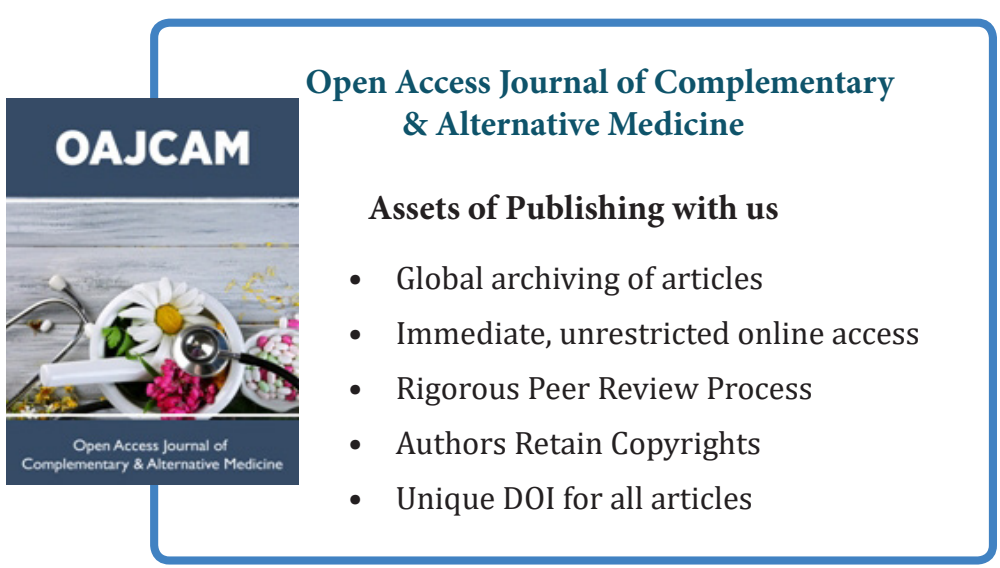

